\title{
PAPER
}

\section{Runaway electron beam control}

To cite this article: D Carnevale et al 2019 Plasma Phys. Control. Fusion 61014036

View the article online for updates and enhancements.

\section{Recent citations}

- Runaway electron synchrotron radiation in a vertically translated plasma

M. Hoppe et al

- Runaway electron seed formation at reactor-relevant temperature

C. Paz-Soldan et al

Kink instabilities of the post-disruption runaway electron beam at low safety factor C Paz-Soldan et al

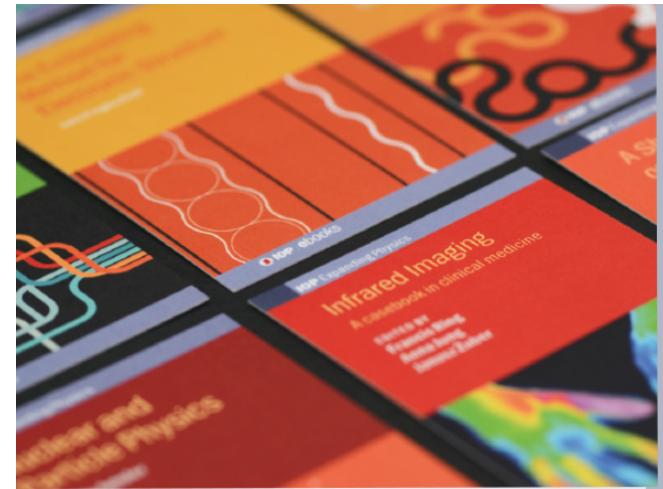

IOP ebooks

Bringing together innovative digital publishing with leading authors from the global scientific community. Start exploring the collection-download the first chapter of every title for free. 


\title{
Runaway electron beam control
}

\author{
D Carnevale $^{1,14}$,, M Ariola $^{9}$, G Artaserse $^{2}$, F Bagnato $^{3}$, W Bin $^{4}$, L Boncagni $^{2}$, \\ T Bolzonella ${ }^{8}$, F Bombarda ${ }^{2}$, P Buratti ${ }^{2}$, L Calacci ${ }^{1}$, F Causa $^{4}(1)$, S Coda ${ }^{3}$, \\ F Cordella $^{2}$, J Decker ${ }^{3}$, G De Tommasi $^{9}$ (i), B Duval ${ }^{3}$, B Esposito ${ }^{2}$, G Ferrò ${ }^{1}$, \\ O Ficker $^{7}$ (1), L Gabellieri ${ }^{2}$, A Gabrielli ${ }^{1}$, S Galeani ${ }^{1}$, C Galperti ${ }^{3}$, \\ S Garavaglia ${ }^{4}$, A Havranek ${ }^{7}$, M Gobbin $^{8}{ }^{(0,}$ M Gospodarczyk ${ }^{1}$, G Granucci ${ }^{4}$, \\ E Joffrin ${ }^{10}$, M Lennholm ${ }^{10}$, A Lier ${ }^{5}$, E Macusova ${ }^{7}$, F Martinelli $^{1}$, \\ J R Martìn-Solis ${ }^{6}$, J Mlynar ${ }^{7}$ (1) , L Panaccione ${ }^{9}$, G Papp ${ }^{5}$, M Passeri ${ }^{1}$, \\ G Pautasso $^{5}$, Ž Popovic ${ }^{6}$, C Possieri ${ }^{1}$, G Pucella ${ }^{2}$, U A Sheikh ${ }^{3}$ (1), \\ G Ramogida ${ }^{2}$, C Reux ${ }^{10}$, F Rimini $^{10}$, A Romano ${ }^{2}$ (1) M Sassano $^{1}$, B Tilia ${ }^{2}$, \\ O Tudisco ${ }^{2}$, D Valcarcel ${ }^{10}$, the FTU team ${ }^{11}$, the EUROfusion MST1 team ${ }^{12}$ \\ and JET Contributors ${ }^{13}$
}

\footnotetext{
${ }^{1}$ Dip. di Ing. Civile ed Informatica, Università di Roma Tor Vergata, Italy

${ }^{2}$ ENEA, Fusion and Nuclear Safety Department, Via E. Fermi 45, I-00044 Frascati, Italy

${ }^{3}$ Ecole Polytechnique Fédérale de Lausanne, Swiss Plasma Center, Lausanne, Switzerland

${ }^{4}$ Istituto di Fisica del Plasma, CNR, Milano, Italy

${ }^{5}$ Max-Planck-Institute for Plasma Physics, D-85748 Garching, Germany

${ }^{6}$ Universidad Carlos III de Madrid, Avda. Universidad 30, E-28911, Madrid, Spain

${ }^{7}$ Institute of Plasma Physics of the CAS, CZ-18200 Praha 8, Czechia

${ }^{8}$ Consorzio RFX, Padova, Italy

${ }^{9}$ Euratom-Enea-Create/Univ. di Napoli Federico II, Via Claudio 21, I-80125, Napoli, Italy

${ }^{10}$ Euratom-CCFE, Culham Science Centre, OX14 3DB, Abingdon, United Kingdom
}

\section{E-mail: daniele.carnevale@uniroma2.it}

Received 6 July 2018, revised 8 October 2018

Accepted for publication 8 November 2018

Published 27 November 2018

\section{Abstract}

Post-disruption runaway electron (RE) beams in tokamaks with large current can cause deep melting of the vessel and are one of the major concerns for ITER operations. Consequently, a considerable effort is provided by the scientific community in order to test RE mitigation strategies. We present an overview of the results obtained at FTU and TCV controlling the current and position of RE beams to improve safety and repeatability of mitigation studies such as massive gas (MGI) and shattered pellet injections (SPI). We show that the proposed RE beam controller (REB-C) implemented at FTU and TCV is effective and that current reduction of the beam can be performed via the central solenoid reducing the energy of REs, providing an alternative/parallel mitigation strategy to MGI/SPI. Experimental results show that, meanwhile deuterium pellets injected on a fully formed RE beam are ablated but do not improve RE energy dissipation rate, heavy metals injected by a laser blow off system on low-density flat-top discharges with a high level of RE seeding seem to induce disruptions expelling REs. Instabilities during the RE beam plateau phase have shown to enhance losses of REs, expelled from the beam core. Then, with the aim of triggering instabilities to increase RE losses, an oscillating loop voltage has been tested on RE beam plateau phase at TCV revealing, for the first time, what seems to be a full conversion from runaway to ohmic current. We finally report

\footnotetext{
11 See the author list of 'Pucella G et al 2014 Proc. 25th IAEA FEC 2014'.

12 See the author list of 'Meyer et al 2017 Nucl. Fusion 57 102014'.

13 See the author list of 'Litaudon X et al 2017 Nucl. Fusion 57102001 '.

14 Author to whom any correspondence should be addressed.
} 
progresses in the design of control strategies at JET in view of the incoming SPI mitigation

experiments.

Keywords: runaways, plasma control system, magnetic confinement

(Some figures may appear in colour only in the online journal)

\section{Introduction}

Post-disruption runaway electron (RE) beams mitigation is one of the main concerns for ITER operations where the RE beam could reach up to $12 \mathrm{MA}$, mainly due to secondary (avalanche) generation mechanisms [1], and even if the RE beam is confined and eventually lost (final loss) with current larger than $2 \mathrm{MA}$, the damages to the vessel can be intolerable for ITER [2].

Joint efforts among researchers all over the world aim at providing mitigation strategies in order to reduce the risk associated with RE beams impacts. The main solution seems to inject heavy particles $(\mathrm{Ar} / \mathrm{Ne})$ before or during the current quench (CQ) that anticipate the post-disruption RE beam formation to limit runaway formation and increase energy dissipation by particle drug and radiation. However, the quantity of injected particles and its assimilation has to induce CQs lasting more than $50 \mathrm{~ms}$ and less than $150 \mathrm{~ms}$ to limit electro-magnetic loads [2]. This operational window seems quite difficult to be obtained; meanwhile, the effectiveness of the secondary (on the fully formed RE beam) MGI injection versus SPI (shattered pellet injection) is under study [3-7]. On the other hand, JET experiments show a reduced RE formation due to the small electrical field at CQ (slower CQ) and plasma contamination thanks to the ITER-like wall [8].

To be able to test mitigation techniques on RE beams, control algorithms have been developed at Tore-Supra [9], DIII-D [5], FTU [10], TCV [11], ASDEX [3] and COMPASS [4]. Indeed, stabilization of the RE beam position and its current is mandatory to have reliable, reproducible and safe experiments. Furthermore, current control to ramp-down the RE beam current can be combined with MGI/SPI dissipation strategies to provide redundancy and backup. The main limitations of RE beam position control for ITER are the poloidal coils and current amplifiers limits: DINA simulations have shown that RE beam control should be effective for CQ drops smaller than 4 MA ( $1 / 3$ of the initial ohmic current). In section 2 we describe the control architecture and the experimental results obtained at FTU and TCV whereas in section 4, are illustrated the proposed strategies to improve the RE beam stabilization at JET for the next SPI campaign.

\section{Control strategy}

We designed control tools to improve RE beam stabilization and ramp-down its current with an architecture conceived to be plugged onto the standard control scheme, i.e. the one that is considered for thermal plasmas. This modular approach allows isolated validation of the new control tools minimizing changes in the code of the standard feedback. The structure of the RE beam controller (REB-C) is shown in figure 1 . The standard (thermal plasmas) control scheme is depicted in black: $\mathrm{C}$ is the regulator used to stabilize the plasma position and current, in many facilities are PID controllers, whereas the block named PLANT represents the dynamics of the electrical circuitry of the active coils, their current amplifiers and the plasma/RE beam dynamics. The block PLANT represents also the sensors (e.g. Mirnov coils) which are involved in the reconstruction of the signals used for plasma position and current stabilization. As discussed in [10], the standard routines that use magnetic measurements to reconstruct the plasma boundaries and its geometric center can be also considered in case of RE beams. Nevertheless, RE beam dynamics are quite different from thermal plasmas and require dedicated control algorithms to improve its confinement as discussed in [12].

The new tools we propose can be identified as three boxes added to the standard control system: the detector of the CQ and RE plateau onset (block D in figure 1), the block $R_{R E}$ that modifies the standard (thermal plasmas) current reference and the block $C_{R E}$ that modifies the desired radial beam center and the output of the controller C. All the aforementioned tools are discussed in detail next.

\subsection{Current Quench and RE plateau detector}

It is necessary to know when the RE beam is formed in order to start a controlled RE beam current ramp-down and introduce new control action by $C_{R E}$. To detect the CQ and the following RE beam formation, i.e. the RE plateau onset, we refer to the discrete time filter and the logics proposed in [13] processing only the $I_{p}$ signal. The filter evaluate an approximate derivative of $I_{p}$ that is used to detect fast $I_{p}$ drops associated to CQs and then the (possible) formation of plateaus characterized by slower current decay. The code implementing the detector is represented by the block D of figure 1 and once the plateau onset is found, the blocks $R_{R E}$ and $C_{R E}$ are then activated to trigger a position controlled current ramp-down. For safety reasons, the ramp-down is also triggered in case the level of hard-X-rays (HXR) overpass a threshold which is associated to high level of interactions between the REs and the vessel. The latter type of ramp-down is called soft-stop.

\subsection{Current ramp-down}

The RE beam current ramp-down is obtained once the block $R_{R E}$ is activated by block $D$, and changes the current reference signal. The current reference is changed as follows: it is initially maintained at the pre-disruption value for a selectable interval of time (10-30 ms) after the plateau onset, then it 


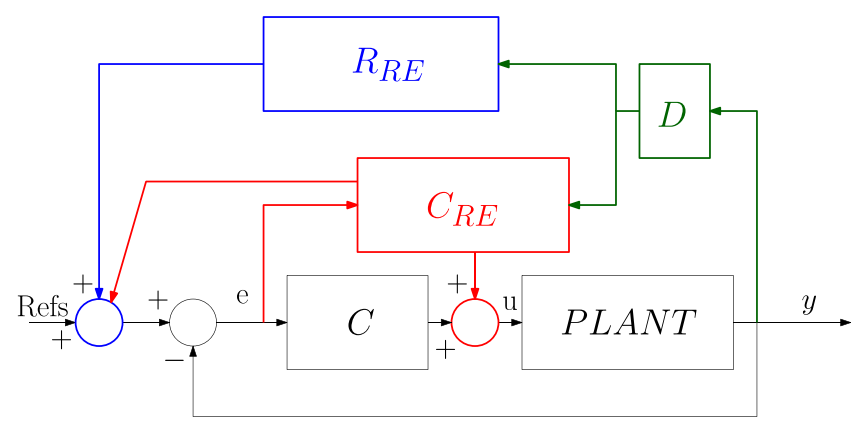

Figure 1. The REB-C scheme: the standard control feedback with the regulator $\mathrm{C}$ is augmented with the block $C_{R E}$ that improves RE beam stabilization, the block $R_{R E}$ that changes the current reference to obtain a ramp-down and the block $\mathrm{D}$ that detects a formed RE beam.

quickly (exponentially) converges to a straight line that goes from the post-disruption (plateau onset) current down to zero with a selectable rate. The current reference is initially (10-30 ms) maintained constant so that the $I_{p}$ control system (part of the block $C$ in figure 1) tries to sustain the current and consequently reduce the plasma/beam radial displacement toward the high-field side (HFS). If the current it is not sustained initially, due to the increased drag caused by impurities at the disruption (CQ), the larger current rate of loss after the CQ induces faster inward (HFS) movement of the plasma/beam column due to unbalanced magnetic forces exerted by PF coils. There is a secondary effect that limits the inner displacement of REs: maintaining constant the current reference a large $I_{p}$ tracking error translates into an increased flux, produced by mean of the ohmic circuit, and such flux is quickly absorbed by REs that increase their energy orbiting consequently with an outer radial center, i.e. REs orbit center moves toward the low-field side (LFS), counteracting the inward shift due to $\mathrm{CQ}$. For the same reason, to avoid too large radial displacements of REs, no more than $30 \mathrm{~ms}$ after the plateau onset the current reference is decreased down to the $I_{p}$ at the plateau onset (postdisruption value), and then ramped down linearly. During the linear ramp-down, the reference is modified in order to limit the loop voltage $\left(V_{\text {loop }}\right)$, measured on-line, below a given predefined threshold. Indeed, experimental analysis have revealed that during the RE beam current ramp-down the higher electrical field the larger MHD modes [4, 11]. Then, the block $R_{R E}$ shifts the desired trajectory toward the measured value in order to reduce the $I_{p}$ tracking error, and then the flux provided by the central solenoid, when $V_{\text {loop }}$ is above a predefined threshold ${ }^{15}$. The shift is obtained changing the parameter $c(t)$ of the current reference $I_{p, \text { ref }}=\operatorname{sign}\left(I_{p, \mathrm{pl}}\right) \max \left(0, c(t)+\left|I_{p, \mathrm{pl}}\right|\left(t_{\mathrm{end}}-t\right) /\left(t_{\mathrm{end}}-t_{\mathrm{pl}}\right)\right)$, where $I_{p, \mathrm{pl}}$ and $t_{\mathrm{pl}}$ are the current and the time at which the ramp-down is triggered, i.e. when a plateau onset is detected by block $D$ or when a soft-stop is request (see section 2.1). The time $t_{\mathrm{end}}$ is such that $-I_{p, \mathrm{pl}} /\left(\mathrm{t}_{\mathrm{end}}-t_{\mathrm{pl}}\right)$ equals the desired ramp-down rate. The value of the parameter $c(t)$ is evaluated on-line to reduce the current error $I_{p}-\mathrm{I}_{p \text {,ref, and then }}$

15 The loop voltage threshold has been set equal to $3 \mathrm{~V}$ for FTU and $1.85 \mathrm{~V}$ for TCV. lowering the $V_{\text {loop }}(t)$, as follows:

$\dot{c}=\left\{\begin{array}{cc}k\left(I_{p}-I_{p, \text { ref }}\right) \operatorname{sign}\left(I_{p, \mathrm{pl}}\right) & \text { if }\left(V_{\text {loop }} \operatorname{sign}\left(I_{p, \mathrm{pl}}\right)>S_{v},\left|I_{p}\right| \geqslant I_{p, \min }\right), \\ 0 & \text { otherwise },\end{array}\right.$

that is such that the $I_{p \text {,ref }}$ converges exponentially, with a time constant $k>0$ chosen larger than the time constant of the ohmic circuitry, to the actual $I_{p}$ in case the measured $V_{\text {loop }}$ would provide too much energy to REs (i.e. when $V_{\text {loop }}$ overpass the selected threshold $S_{v}$ ). It has to be noted that the measured $V_{\text {loop }}$ does not depend only on the flux provided by the central solenoid but also on the current decay, MHD activity and drag $\left(Z_{\text {eff }}\right)$ but the algorithm, at the present stage, does not take these elements into account.

\subsection{Position control}

The position control block $C_{R E}$ in figure 1 has been designed to improve stabilization of the RE beam. The magnetic measurements are currently used in different tokamaks (DIII-D, FTU, TCV, ASDEX, JET, KSTAR) to estimate the beam position: experiments at DIII-D analyzing soft-X camera and the scanning $\mathrm{CO}_{2}-\mathrm{CO}$ interferometer at FTU have shown that such approximation is valid $[10,14]$. We intend to provide a refinement of the standard stabilization system in $C$ to improve tracking performances and, consequently, safety for RE beam experiments. The block $C_{R E}$ input the position tracking error as the controller $C$. $C_{R E}$ redefines the desired radial position of the beam center and modifies the request of current (FTU) or voltage (TCV) to the PF coils requested by $C$. The reference of the radial coordinate of the beam center is moved toward the HFS, as suggested in [10, 15], to reduce RE-vessel interactions: the radial center reference is reduced linearly to a desired value (about 15\% smaller than the standard one). Indeed, with respect to standard plasmas, the radial center of the REs orbits is shifted toward the LFS due to their higher energy [15].

2.3.1. Fast hybrid controller. To improve fast displacements recovery an ad-hoc tool has been introduced exploiting the framework of hybrid systems [16-18]. We provide a quick overview of the fast controller: let $e$ be the (vertical/radial center) tracking error and $E=(e, \dot{e}, \ddot{e})$ the state of the position error system. The new controller acts generating control signal as ramps whenever $E$ enters into an unsafe region $\Omega$ in order to steer $E$ back into the safe one. Vertical and radial displacements of the RE beam are considered to be decoupled and then two independent fast controllers acting on the PF coils have been designed.

Define the set $\Omega$ as

$$
\begin{aligned}
\Omega= & \left\{E \in \mathbb{R}^{3}:|e|>\sigma_{1} \wedge|\dot{e}|>\sigma_{2} \wedge e \dot{e}\right. \\
& \left.>0 \wedge \operatorname{sign}(e) \ddot{e}>\sigma_{3}\right\},
\end{aligned}
$$

for given parameters $\left(\sigma_{1}, \sigma_{2}, \sigma_{3}\right)$ and where the time derivatives of the tracking error signal $e$ are evaluated online via specific filters [13]. Although it is not immediate, the set $\Omega$ identifies a portion of the state space in which trajectories are pointing far from the origin, i.e. the tracking error is going to increase immediately after $E$ enters in $\Omega$. As 

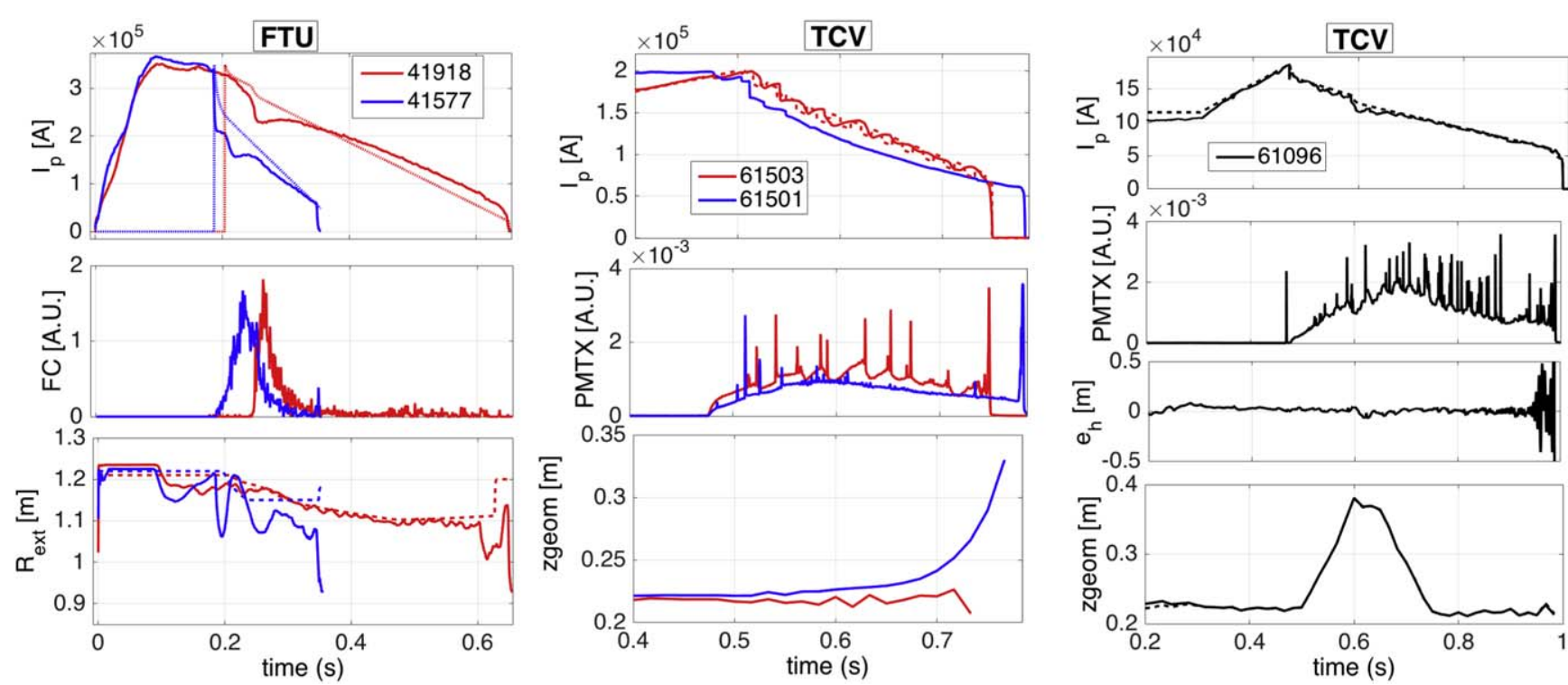

Figure 2. The REB-C performances: (left) FTU controlled ramp-down with (\#41918) and without (\#41577) the fast controller. (center) TCV controlled ramp-down with (\#61503, also with current oscillations) and without (\#61501) the REB-C that is used to obtain (right) a large RE beam vertical displacement of about $11 \mathrm{~cm}$, shot \#61096.

an example, consider the condition $e \dot{e}>0$ in (2): if $e$ and $\dot{e}$ are positive then $e$ will (instantaneously) further increase, or if both $e$ and $\dot{e}$ are negative then $e$ will (instantaneously) further decrease. The output $u_{f}(t)$ of the fast controller is generated via the following equations (hybrid system):

$$
\begin{gathered}
u_{f}(t)=-\operatorname{sign}(e(t)) k_{r}(t)\left(t-t_{k}\right), \text { if } E \in \Omega, \\
\dot{u}_{f}(t)=-k_{d} u_{f}(t), \text { if } E \notin \Omega,
\end{gathered}
$$

where $t_{k}$ is the most recent time at which $E(t)$ entered into $\Omega$. The fast controller has been designed initially to cope with elongated unstable discharge at FTU [19] due to the phase lag introduced by the current amplifier, not designed to deal with vertical instability since FTU is a tokamak with circular plasmas. Then, to manage this phase lag in the control loop, the fast controller (3)-(4) exploits the second derivative $\ddot{e}$ to retrieve preemptive information on the effect of the ramp steering $E$ back into the safe region: this information is contained in the constraint $\operatorname{sign}(e) \ddot{e}>\sigma_{3}$ of (2). Indeed, selecting for example $\sigma_{3}$ negative, the ramp is switched off exponentially by (4) if sign (e) $\ddot{e}$ is negative, meaning that the error derivative is going to decrease and, due to the phase lag, $E(t)$ might soon leave $\Omega$ and the control action needs to be reduced to avoid instability: phase lag usually introduce unstable oscillations. The origin of the overall closed-loop system can be proved to be asymptotically stable assuming a linear transfer function of the plant, with parameters within a bounded region, recurring to the stability analysis tools for hybrid systems [16]. The slope of the ramp $k_{r}(t)$ is changed adaptively by an heuristic: it is increased when $E(t)$ enters into $\Omega$ on the same 'direction' (sign of $e \dot{e}$ ) for a certain number of times within a predefined interval of time, whereas $k_{r}(t)$ is decreased when oscillations are detected since they might be caused by high values of $k_{r}$. Furthermore, to avoid fast $u_{f}$ oscillations on $\Omega$ boundaries (chattering), an hysteresis is implemented.

This control action does not excite fast plant dynamics unlike bang-bang type controllers and its parameters have been experimentally tuned very quickly thanks to the adaptive gain $k_{r}$ (3 shots for FTU and 1 for TCV). The fast controller did not only improve the RE beam control but also vertical stability for FTU elongated plasma discharges [19].

The performances of the new control tools are shown in figure 2. The left column shows pictures from FTU shots: \#41918 is with an advanced version of REB-C also having the fast controller that was missing in \#41577. The middle column shows TCV pulses with REB-C \#61503 and without \#61501: the standard controller can in about 50\% of the shots maintain the RE beams, whereas REB-C has never shown a vertical instability like in \#61501. Furthermore, the stabilization of the shot \#61503 with the REB-C controller is harder since a square wave is added to the $I_{p}$ reference to induce electrical field oscillations: such current oscillation increases the MHD activity, enhances RE losses ${ }^{16}$ and deteriorates radial displacements. Nevertheless REB-C outperforms the standard controller. The right column depicts an RE beam desired vertical displacement of about $11 \mathrm{~cm}$ with satisfactory tracking error $e_{h}(t)=$ zgeom $_{\text {ref }}$-zgeom $(t)$. The signal zgeom is the vertical coordinate of the RE beam center provided by LIUQE equilibrium reconstruction code [20]. In figure 2, the time traces in the $I_{p}$ plot are the plasma/RE beam current (solid) and the current reference $I_{p \text {,ref }}$ (dashed). For FTU, we show the counts (multiply by the factor 1E14) of the fission chamber (FC) camera sensible to photo-fissions induced by REs with energy higher than $6 \mathrm{MeV}$ hitting the vessel, whereas for TCV, the HXR counts of the photo multiplier (PMTX) tube measuring emissions of runaways (synchrotron and collisions) are shown. At the bottom plots of figure 2, for FTU we show the estimated external radius of the beam center $R_{\text {ext }}$ (solid) and the desired one (dashed). For TCV, the vertical tracking error $e_{h}$ and the vertical coordinates of the

\footnotetext{
16 Similar results where electrical field oscillations have induced RE losses have been discussed in [21] and [4].
} 

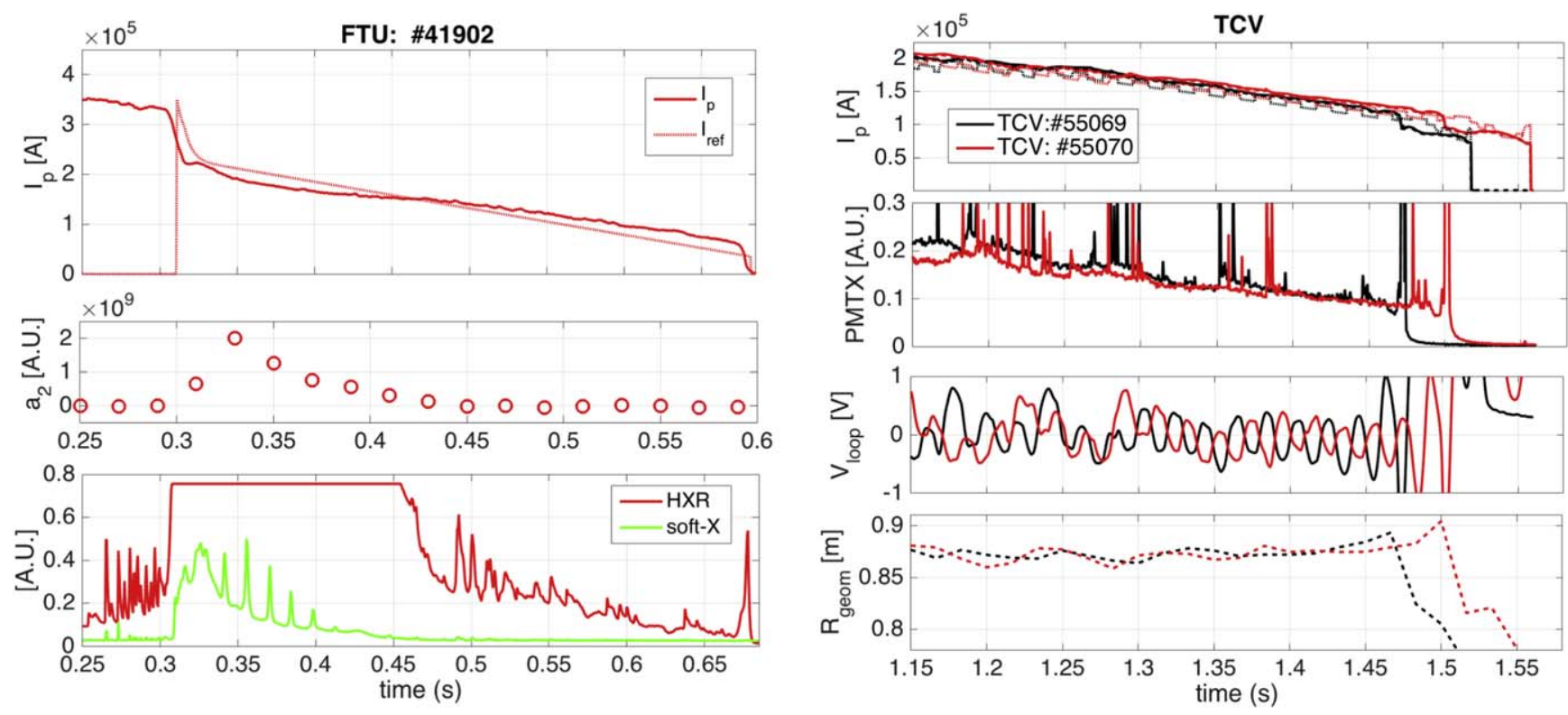

Figure 3. Right: an FTU RE beam current ramp-down showing that the coefficient $a_{2}$, fitting the REs synchrotron radiation spectra, decreases after current quench proving that RE energy is reduced, also coherently to HXR. Left: two shots with oscillating loop voltages during rampdown showing complete loss/conversion of REs before the final current loss.

beam center (zgeom) are shown. It has to be noted that the loss of RE beams at TCV, when the REB-C is used, is due to a delay introduced by the PF current amplifiers at zero crossing: we are studying possible solutions to this issue.

The RE energy suppression during the current ramp-down is confirmed by the RE and image spectroscopy (REIS) [10], a diagnostic that provides the spectra of the synchrotron radiation emitted by in-flight REs: after the plateau onset the RE energy distribution peak, which is correlated with the value of the coefficient $a_{2}$ of a second order polynomial $a_{2} x^{2}+a_{1} x+a_{0}$ fitting the measured spectra, moves toward lower energies, according also to other diagnostics such as HXR, FC, proving that RE energy is decreased during the ramp-down.

\subsection{Final loss}

RE beams can provoke potential damage at the final loss due to magnetic to kinetic energy conversion, as discussed in [22-24]. Analysis on FTU discharges have revealed, during RE current ramp-down, anomalous fast radial inward movements of the $\mathrm{RE}$ beam associated to partial/small RE loss against the vessel, typically within 150-180 ms after the onset of the ramp-down (compared to the external radius of the beam in figure 2 approximately at $0.6 \mathrm{~s}$ ). At TCV two RE beam ramp-down adding electrical field oscillations (square waves added to the $I_{p}$ reference) have shown an extremely interesting phenomenon: before the usual RE beam final loss the REs seem to disappear whereas the current remains almost unchanged, as if an ohmic plasma with no runaways forms, with a reduced $L_{i}$ and a sudden inner radial displacement. The inner radial displacement when the REs are loss at TCV can be correlated, as in FTU, to the sudden loss of REs energy. To give a possible explanation to such findings we can refer to the hysteresis that has been found to affect the REs generation/suppression [25-27]. In the right plots of figure 3, before the pulses end, runaways are lost completely but there is only a $15 \%$ current drop: the electrical field oscillations might have induced the (sudden) hysteresis transition across the unstable equilibria that divide the ohmic from the runaway region in the energy state space representation. Temperature measurements exclude the possibility that a thermal plasma would coexist with REs before their loss. Further experiments have been conducted with larger $V_{\text {loop }}$ oscillations with the aim of obtaining similar preemptive loss of REs without current drops, i.e. a possible formation of ohmic plasma from a RE beam. However, in these new experiments, one of which is the shot \#661502 in figure 2, the plasma conditions changed and the mean electrical field during the ramp-down resulted in being slightly positive (higher drag due to larger amount of impurities injected by the new disruption mitigation valve system), possibly inhibiting a gradual REs energy reduction process. Further experiments are planned to investigate this important feature that would possibly allow to reduce the issue of the RE energy conversion into kinetic one at the final loss.

\section{Deuterium pellets and laser blow-off injections on FTU}

The REB-C has been used to stabilize the RE beam position and perform experiments on deuterium pellets and laser blowoff injections into flat-top plasma current with REs and postdisruption RE beams, respectively. Indeed, as mentioned in the introduction, the RE beam position and current control system can the be coupled with other mitigation techniques or can be used to asses their effectiveness, providing experimental repeatability and improving safety.

The FTU facility is provided with a horizontal pellet injector that can launch, triggered by a TTL signal of the 

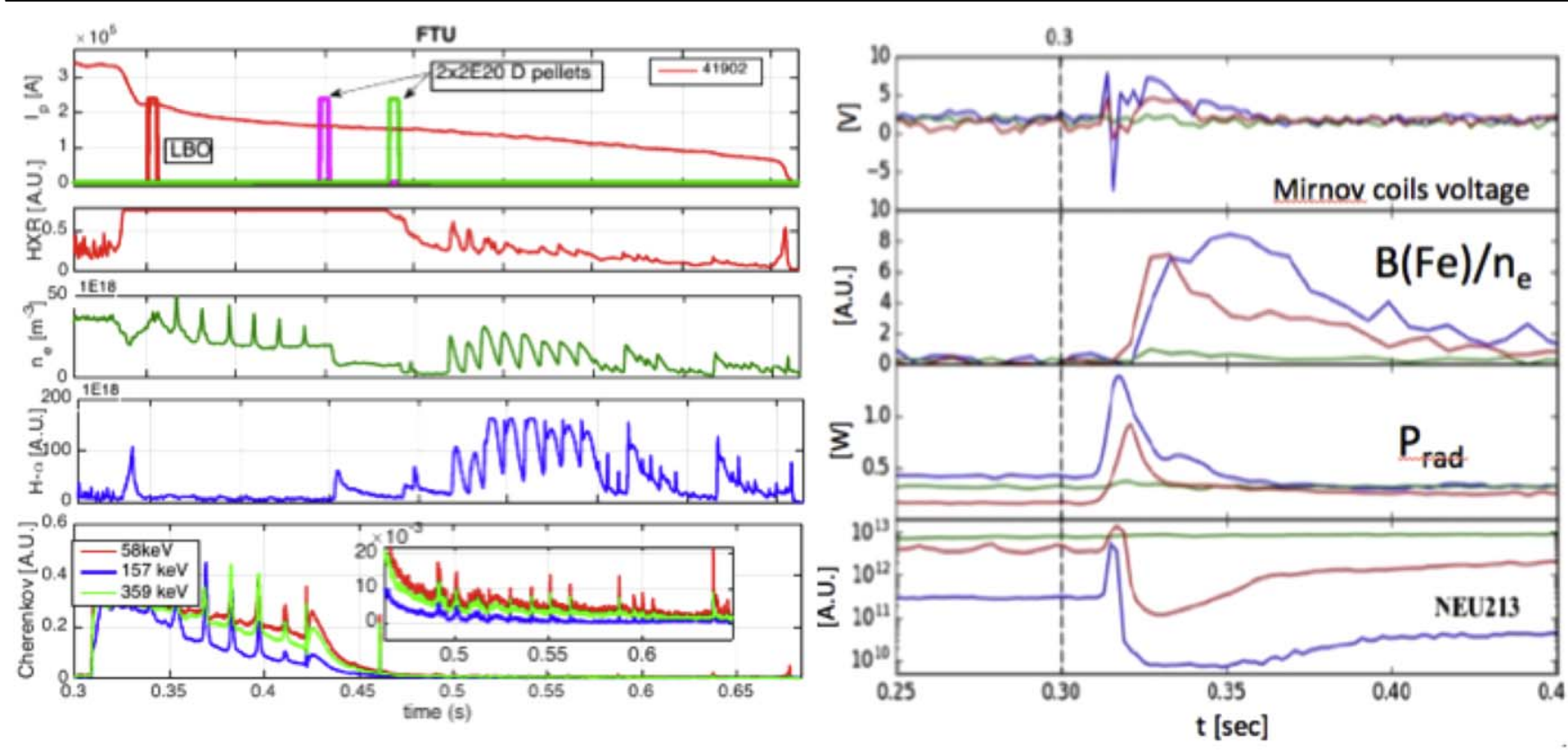

Figure 4. Left: $\mathrm{D}_{2}$ pellets and LBO injections on a post-disruption RE beam showing that the density drops at pellets injection while instabilities sets in after the plateau onset expelling REs out of the core as detected by the Cherenkov probes. Right: LBO injection of iron on flat-top plasmas with in-flight REs causes small disruptions expelling REs, as revealed by the drops of NEU213, proportionally to the Iron ionization $\left(\mathrm{B}\left(F_{e}\right) / n_{e}\right)$

control system, up to two 'small' deuterium pellets of about $1 \times 10^{20}$ atoms at a speed of $1200 \mathrm{~m} \mathrm{~s}^{-1}$ and two 'large' pellets with $2 \times 10^{20}$ atoms at $1000 \mathrm{~m} \mathrm{~s}^{-1}$. The time to reach the core of a normal plasma is about $0.3 \mathrm{~ms}$ and is usually used for fueling increasing the density up to $8 \times 10^{20}$ with $I_{p}=1.2 \mathrm{MA}$ (8T) [28]. To have an idea of the amount of atoms injected with respect to the plasma volume, a large pellet if injected on a $360 \mathrm{kA}(5 \mathrm{~T})$ discharge induces a density limit disruption.

Fast H-alpha detectors, Mirnov coils (MHD sensors), a fast $(65 \mu \mathrm{s}) \mathrm{CO}-\mathrm{CO}_{2}$ scanning interferometer and $\mathrm{X}-\mathrm{VUV}$ spectrometer Schwob are used to analyze the discharges.

The main results of $D_{2}$ pellets and Laser Blow-Off (LBO) [29] injections are the following:

- Flat-top: a small pellet yields $n_{e}$ increase and with almost the same probability (50\%) can lead to RE total expulsion (MHD driven instabilities) or RE increase (due to the increase of the electrical field). LBO can cause interesting RE expulsion, as for pellets, but do not increase the RE number as shown in the right plots of figure 4 . The amplitude of the induced MHD activity expelling the REs from the core seem to be proportional to the ionization of the impurity detected by the Schowb spectrometer as shown in the time trace of the second plot (right column) representing the evolution of the $\mathrm{F}_{e}$ XXIII (135.80 A) line brightness normalized with respect to $\mathrm{n}_{e}$, whereas the time traces NEU213 are $\gamma$ rays and neutrons counts, measured by a scintillator, and provide qualitatively the number of in-flight REs. It is evident that the drops of NEU213 few milliseconds after the LBO injection at time $0.3 \mathrm{~s}$, revealing that REs are lost, is correlated with the level of ionized atoms $\left(\mathrm{B}\left(\mathrm{F}_{e}\right) / n_{e}\right.$ in the second plot $)$ and radiated power $\left(P_{\mathrm{rad}}\right)$. It is clear indeed that the more Fe atoms are ionized, the more they interact with the plasma triggering an instability expelling REs. It has to be noted that, for the shot whose signals are depicted in red, after the REs expulsion the other REs are generated given that was a low density discharge.

- RE beam: pellets induce a further decrease of the temperature, which is already low, so that recombination takes place and electron density drops. Only in one of the discharges did we record a large density increase when the pellets have been injected far from the plateau onset: the background plasma might have gained energy, then temperature, during the ramp-down. LBO does not provide any effect due to the low temperature of the beam (no ionization). Instabilities expelling REs have been recorded during the ramp-down as shown by MHD and Cerenkov probe spikes clearly visible in the right plots of figure 4. Fan instability [30] sets in after $0.45 \mathrm{~s}$ and has been recognized by marks on ECE emission, whereas the instability right after the CQ up to $0.45 \mathrm{~s}$ is different. Recent studies might suggest that such losses are indication of whistler waves [31].

\section{JET control tools}

Past experiments on JET facilities have shown that selecting specific initial position $(z=0.01, r=0.4)$ of a limiter low density circular plasma, the standard control system allowed to maintain a beam up to $200 \mathrm{~ms}$ as shown in figure 5 . Nevertheless, large vertical excursions leading to premature loss of the RE beam have been observed. In view of the next 

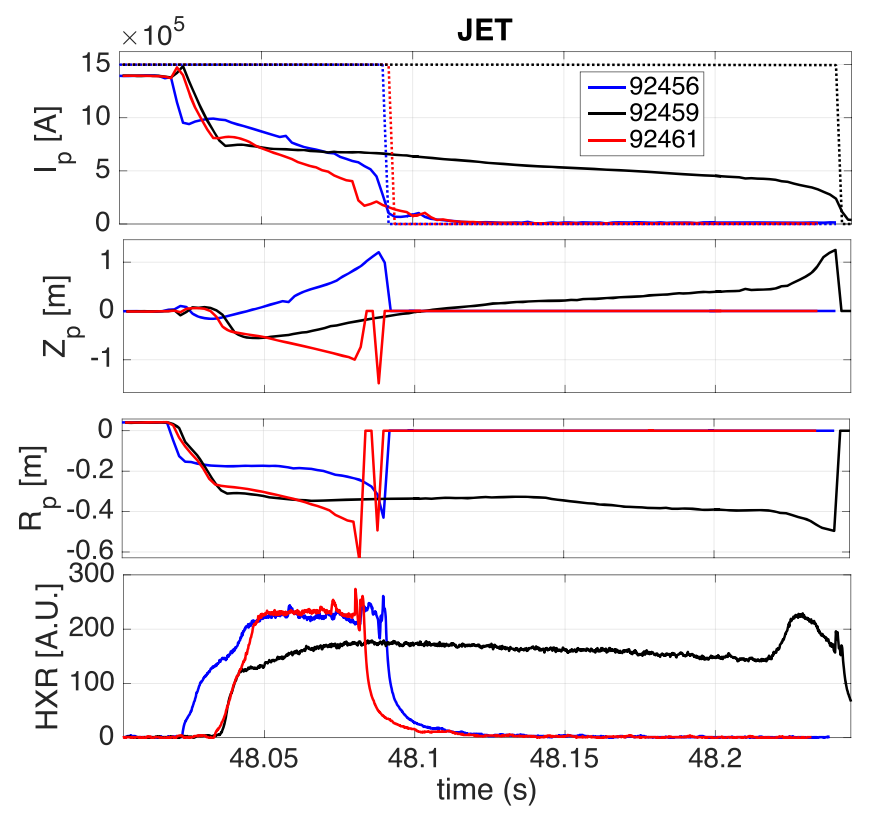

Figure 5. JET past experiments: the standard control system has shown to be able to confine the RE beam up to $200 \mathrm{~ms}$ (\#92459) although large vertical displacements usually lead to premature RE beam losses.

shattered pellet injections (SPI) experiments to study dissipation effects on REs energy, it is mandatory to have an improved and reliable RE beam confinement in order to have experiments repeatability. Analysis on RE beam losses revealed that the controller VS5, designed to stabilize the fast vertical plasma displacements (VDE), does not react to the slow drift which characterizes the movements of the RE beam plateau phase and leads to premature losses of the beam, as shown by the vertical center of the beam $Z_{p}$ in figure 5 . Indeed, the velocity observer that estimates VDEs acts as an high-pass filter and then slow drifts are filtered out. Consequently, the vertical velocity feedback loop feed with the observer output does not react to slow drift. It comes naturally then, since changes to the code of the position controller have not begin allowed, to modify the observer to make it sensible to slow drifts, without loosing the capability of the standard one to sense fast movements. Such an observer can be designed by properly selecting the parameters of the linear difference equation (linear time invariant system) already implemented in the real-time code: we need only to provide different configuration parameters to design the new observer and the real-time control code is not changed. However, we desire to use the new observer only in case of RE beams plateau, then we implemented the CQ and plateau detector algorithm in order to switch from the standard to the new observer once the CQ is detected.

Apart of the new observer design, it is possible to activate also the the JET shape controller (SC) [32] at the plateau onset, that was switched-off in the past experiments. The SC might help in maintaining the vertical/radial stability of the beam since it can be configured to stabilize in closed loop the vertical $Z_{p}$ and radial $R_{p}$ coordinates of the beam center (estimated by equilibrium code). However, the response time of the SC in the feedback configuration is too slow for the RE beams. To provide faster responsiveness of the SC a possible solution consists into providing directly feed-forward currents of the PF coils used by the SC (P4 imbalance coils), i.e. the $\mathrm{SC}$ tries to impose directly the desired current on the PF coils instead of closing the feedback on the beam position. To provide a possible strategy for the selection of such preprogrammed coils current, a new tool similar to the rapidly-exploring random tree (RRT) [33] used in robotics has been conceived. Starting from experimental data, a graph representing plant transitions induced by given control actions is constructed and updated at each new experiment. The nodes of the graph are the state of the plant, the vector $\left(Z_{p}, \dot{Z}_{p}, \ddot{Z}_{p}\right)$ in this case, and the arches that allows to pass from node to node are the experimental control inputs (PF coils currents). At each node an LTI system is identified using standard identification tools. The control problem is then recast into an optimization path planning problem and a number of optimization tools can be used. Then, shot by shot, the system will suggest to the RDOs the pre-programmed currents that could improve the RE beam stabilization. This approach provides feed-forward signals and it is not robust by definition, although faster than the SC in closed loop and might steel provide help if experiments are repeatable. Again, this solution has been proposed to bypass real-time control code changes.

\section{Conclusion}

The new control tools tested at FTU and TCV have shown that the RE beam position can be stabilized and its current ramped-down. RE beam confinement improved with the the installation of the REB-C at FTU and TCV, as shown by radial and vertical position of the RE beam center in figure 2 . Given that the Hybrid Fast Controller is model-free, it is intrinsically robust and the portability from FTU to TCV paves the way to ITER implementation. It might be possible to further improve the REB-C designing a dedicated $I_{p}$ controller, without relying on the standard one, possibly including real-time measurements of $Z_{\text {eff }}$ to better regulate the flux absorbed by REs. Concerning the current ramp-down, it has been confirmed that controllability of the RE beam is dependent on the $I_{p}$ decaying rate: mitigation techniques should be compliant with the rate at which controllability is lost. Note that if fast RE current reduction is requested, due to limitations on the current rate of the central solenoid, a combined action of MGI/SPI is necessary. The other way around to slow the decaying rate, almost no flux has to be provided by the central solenoid and controllability improves: current on the PF coils has to be changed to a lower rate (assuming no VDEs). At TCV, large RE beam vertical displacements have been successfully achieved and a new interesting phenomenon has been observed: REs are suddenly replaced by ohmic plasma, opening a possible new way to treat the final loss issue. On FTU, thanks to the RE beam control system, repeatable deuterium pellets experiments have been performed showing that $\mathrm{D}$ pellets do not increase REs 
dissipation rate (RE beams not formed using MGI), and large instabilities expelling REs have been observed. LBO experiments have shown that small disruptions leading to important $\mathrm{RE}$ loss can be achieved using iron on flat-top low density discharges. This might suggest to use LBO before MGI to see if post-disruption RE current can be lowered. Finally, we have briefly discussed the observer redesign at JET and the use of a new tool based on graphs to possibly improve control performances for the next SPI experiments.

\section{Acknowledgments}

This work has been carried out within the framework of the EUROfusion Consortium and has received funding from the Euratom research and training programme 2014-2018 under grant agreement No 633053. The views and opinions expressed herein do not necessarily reflect those of the European Commission.

\section{ORCID iDs}

D Carnevale (1) https://orcid.org/0000-0001-6214-7938

F Causa (i) https://orcid.org/0000-0003-0508-3100

G De Tommasi (i) https://orcid.org/0000-0002-8509-7176

O Ficker (ib https://orcid.org/0000-0001-6418-9517

M Gobbin (1) https://orcid.org/0000-0001-5261-3683

J Mlynar (10 https://orcid.org/0000-0003-4718-4321

U A Sheikh (1) https://orcid.org/0000-0001-6207-2489

A Romano (i) https://orcid.org/0000-0002-1228-6005

\section{References}

[1] Connor J W and Hastie R J 1975 Relativistic limitations on runaway electrons $\mathrm{Nucl}$. Fusion $\mathbf{1 5} 415$

[2] Lehnen M et al 2015 Disruptions in ITER and strategies for their control J. Nucl. Mater. 46339
[3] Papp G et al 2016 Preprint: 2016 IAEA Fusion Energy Conf. [EX/9-4] WPMST1 - CP(16) 15157

[4] Ficker O et al 2017 Nucl. Fusion 57076002

[5] Hollmann E M et al 2013 NF 53083004

[6] Shiraki D et al 2018 Nucl. Fusion 58056006

[7] Gál K et al 2008 Plasma Phys. Control. Fusion 50055006

[8] Reux C et al 2015 Nucl. Fusion 55129501

[9] Saint-Laurent F et al 2011 Proc. 38th EPS Conf. Plasma Physics vol O3 p 118

[10] Esposito B et al 2016 Plasma Phys. Control. Fusion 59 014044

[11] Carnevale D et al 2017 44th EPS P 1.152

[12] Gospodarczyk M et al 2018 FED 135 (A) 42-9

[13] Boncagni L et al 2013 A first approach to runaway electron control in FTU Fusion Eng. Des. 81109

[14] Eidietis N W et al 2012 Phys. Plasmas 19056109

[15] Hollmann E M et al 2013 Nucl. Fusion 53083004

[16] Goebel R, Sanfelice R G and Teel A R Hybrid Dynamical Systems Modeling, Stability, and Robustness (Princeton, NJ: Princeton University Press)

[17] Carnevale D et al 2016 Int. Journal of Adaptive Control and Signal Processing (New York: Wiley) https://doi.org/10. $1002 /$ acs. 2679

[18] Carnevale D, Galeani S, Menini L and Sassano M 2016 IEEE Trans. Autom. Control 6 2416-31

[19] Ramogida G et al 2017 Nuclear Materials and Energy 12 1082-7

[20] Moret J M et al 2015 Fusion Eng. Design 91 1-5

[21] Carnevale D et al 2016 EX/P8-22 (IAEA)

[22] Putvinski S et al 1997 Plasma Phys. Control. Fusion 39 B157

[23] Riemann J et al 2012 Phys. Plasmas 19012507

[24] Martìn-Solìs J R et al 2014 J.R. Nucl. Fusion 54083027

[25] Carnevale D et al 2015 42nd EPS Conference on Plasma Physics P4.192

[26] Popovic̀ Ž et al 2016 Phys. of Plasmas 23122501

[27] Martìn-Solìs J R et al 2000 Phys. of Plasmas 73814

[28] Frigione D et al 2001 Nucl. Fusion 411613

[29] Romano A et al 2018 47th EPS P5.105 3

[30] Vlasenkov V S, Leonov V M, Merezhin V G and Mukhovatov U S 1973 Nucl. Fusion 13509

[31] Liu C et al 2016 45th EPS P2.4005

[32] Maviglia F et al 2015 FED 96-97 668-71

[33] LaValle S M and Kuffner J J 2000 Proc. Workshop on the Algorithmic Foundations of Robotics

[34] Causa F et al 2015 Nucl. Fusion 55123021

[35] Boncagni L et al 2015 IEEE Conf. on Decision and Control (https://doi.org/10.1109/CDC.2015.7402553) 